\title{
Evaluation of electrical resistivity, residual stress and surface roughness of sputtering indium tin oxide films with different thicknesses
}

\author{
Chuen-Lin Tien ${ }^{1 *}$, Tsai-Wei Lin ${ }^{1}$, Shu-Hui $\mathrm{Su}^{2}$ \\ ${ }^{1}$ Department of Electrical Engineering, Feng Chia University, \\ Taichung, Taiwan \\ ${ }^{2} \mathrm{Ph}$.D. Program of Electrical and Communications Engineering, \\ Feng Chia University, Taichung, Taiwan
}

${ }^{*}$ Corresponding author: cltien@fcu.edu.tw

\begin{abstract}
This paper investigates the influence of film thickness on the electrical and mechanical properties of transparent indium tin oxide (ITO) thin films. Two groups of ITO thin films deposited on unheated substrates were prepared by the radio-frequency magnetron sputtering technique. The biaxial residual stress and surface roughness for two groups of ITO thin films were measured by a Twyman -Green interferometer and a Linnik microscopic interferometer, respectively. The electrical resistivity of the ITO films was measured by a four-point probe apparatus, the thickness was determined mechanically with a profilometer. The measurement results show that the average resistivity of ITO thin films decreases with increasing the deposited thickness. The compressive residual stress in the ITO thin films decreases with increasing the deposited thickness. We also find that an anisotropic stress in the two groups of ITO films is more compressive in a certain direction. The RMS surface roughness in the two groups of ITO films is less than $1 \mathrm{~nm}$.
\end{abstract}

Keywords: indium tin oxide, magnetron sputtering, residual stress, surface roughness.

\section{Introduction}

Transparent conductive thin films have been widely applied in electronics, optoelectronics and solar cells fields. Indium tin oxide (ITO) is one of the most widely used transparent conducting oxides due to its low resistivity and excellent optical transparency [1-4]. ITO thin films can be prepared by various deposition techniques, such as thermal evaporation $[\underline{5}, \underline{6}]$, radio-frequency $(\mathrm{RF})$ magnetron sputtering $[\underline{7}, \underline{8}]$ direct current (DC) magnetron sputtering [9-12], plasma ion-assisted evaporation [13], activated reactive evaporation [14], and sol-gel method [15]. The RF sputtering method 
is the most commonly used among all these methods because of the more compact structure and good transmittance of thin films prepared by this technique.

In recent years, the engineering applications of ITO thin films have become an important issue. Many studies focusing on the optimized process parameters and their electrical properties have been carried out, but there has been a lack of study on the relationship between film thickness, biaxial anisotropic stress and surface roughness. Measuring and controlling residual stress in thin films are a critical challenge in optical precision devices development. In general, optical thin film processing results in residual stress which may cause buckling of the thin films or deformation of the substrate. Evaluation of the residual stress is very important for thin film coatings. The aim of this paper is to evaluate anisotropic stress, root-mean-square (RMS) surface roughness and electrical resistivity in ITO thin films. The difference of residual stress and surface roughness of ITO films with different deposited thickness was compared between the two experimental sets with the same deposition parameters. Therefore, we have analyzed the residual stress by the fast Fourier transform (FFT) method [16], and used an interferometric microscopy to determine the surface roughness of thin films. Microstructure of ITO thin films was examined by means of field-emission scanning electron microscopy (FESEM; Hitachi S-4800).

\section{Experimental details}

In this study, thin films were prepared by the radio frequency (RF) magnetron sputtering method. The surfaces of the substrates were cleaned before the application of coating. The silicon and glass substrates were cleaned successively in acetone, alcohol, and distilled water with an ultrasonic cleaner for $15 \mathrm{~min}$, after which they were dried in a vacuum dryer for $5 \mathrm{~min}$ at room temperature. ITO thin films were deposited on B270 glass substrates by using RF magnetron sputtering technique. ITO thin films were grown in a high vacuum deposition system. The ceramic ITO target was arranged directly below the substrate. A RF magnetron sputtering system equipped with RF power suppliers is shown in Fig. 1. The target for the deposition was a 2-inch disk of $\mathrm{In}_{2} \mathrm{O}_{3}-10 \mathrm{wt} . \% \mathrm{SnO}_{2}$ oxide target. The vacuum chamber was evacuated to $6.65 \times 10^{-3} \mathrm{~Pa}$. The reaction gas was an argon (Ar) and oxygen $\left(\mathrm{O}_{2}\right)$ mixture. The argon flow rate was $20 \mathrm{sccm}$, and the oxygen flow rate was in the range of $1-5 \mathrm{sccm}$. RF magnetron sputtering power and sputtering pressure were $100 \mathrm{~W}$ and $0.4 \mathrm{~Pa}$, respectively. The distance between the substrate and the target was about $75 \mathrm{~mm}$. The target was pre-sputtered for $10 \mathrm{~min}$. The film thickness was controlled by quartz crystal monitoring. ITO thin films with three different thicknesses of 200, 300 and $350 \mathrm{~nm}$ were deposited on B270 glass substrates.

The characteristics of the biaxial residual stress and surface roughness of ITO thin films with three different thicknesses were investigated experimentally. The average residual stress and anisotropic stress in ITO thin films with different deposition thicknesses was measured by a Twyman-Green interferometer, as shown in Fig. 2. As can 


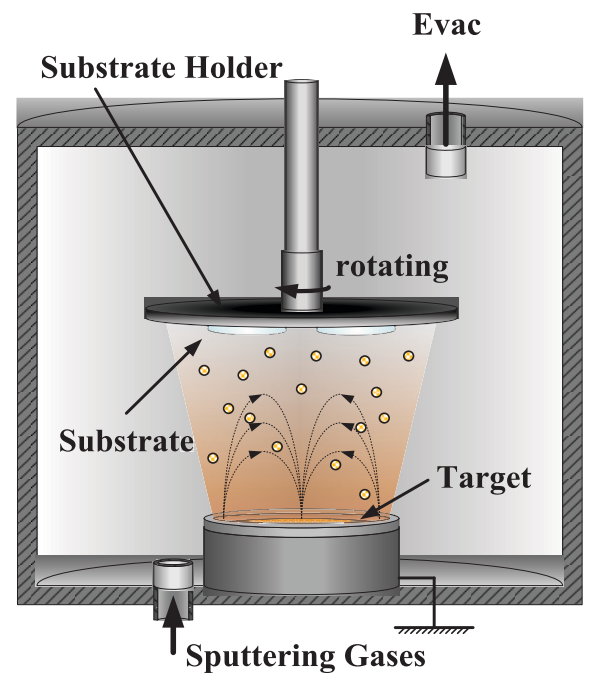

Fig. 1. The radio frequency (RF) magnetron sputtering system.

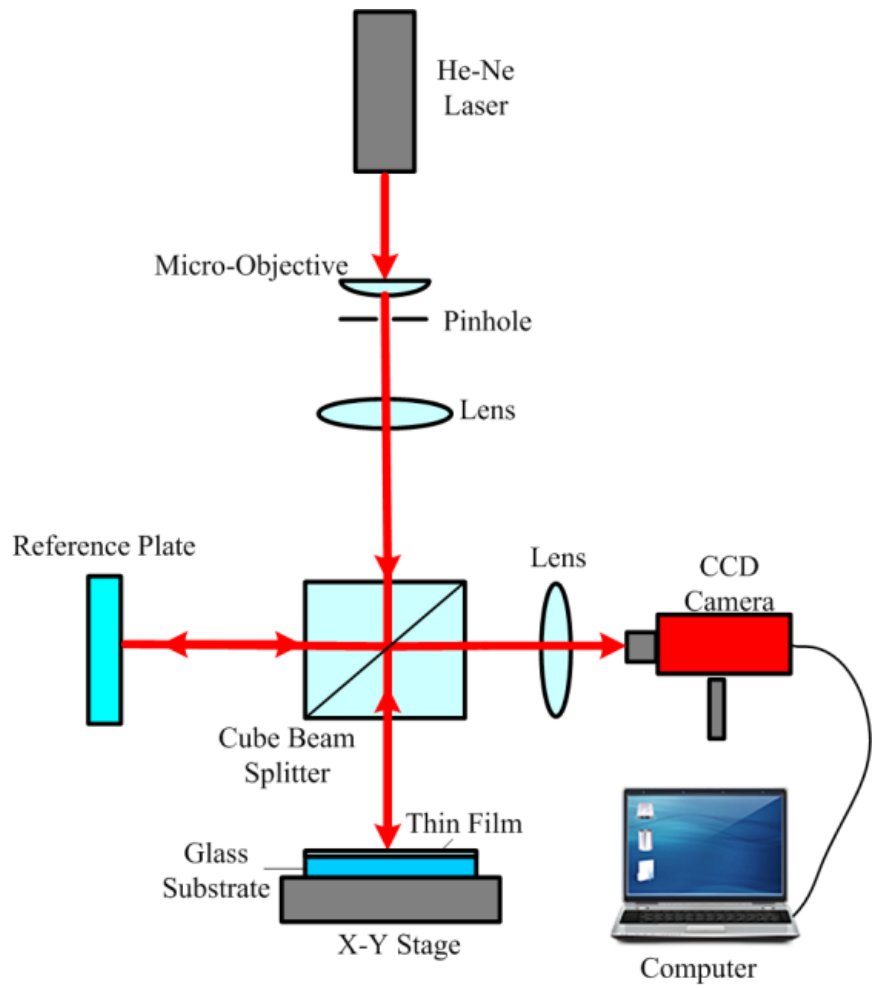

Fig. 2. System for the measurement of thin film stress. 
be seen in this figure, the light source is a helium-neon laser with a center wavelength of $632.8 \mathrm{~nm}$. The laser light source is incident through a micro-objective lens and a pinhole, usually called a spatial filter, and then forms a point light source. The point light source is originally converted into a uniform parallel beam by a collimating lens of appropriate focal length. When the parallel beam hits the beam splitter, it splits the parallel beam into two beams. One beam is reflected by a reference plate surface, while the other beam will be transmitted to the film surface of the tested substrate. Two beams are reflected to the beam splitter and then overlap to form interference fringes on a screen. We use a CCD camera to capture the interference fringes imaged on the screen. The interference fringe pattern is analyzed by self-developed MATLAB software program. The stress analysis algorithm is based on the fast Fourier transform (FFT) method $[17,18]$. The thickness of ITO films was measured by using an alpha-step profilometer.

The proposed method is a non-contact and non-destructive measurement to evaluate the residual stress of thin films. In general, the residual stress of ITO thin films can be expressed in terms of the modified Stoney's formula [19]:

$$
\sigma=\frac{1}{6} \frac{E_{\mathrm{s}}}{1-v_{\mathrm{s}}} \frac{t_{\mathrm{s}}^{2}}{t_{\mathrm{f}}}\left(\frac{1}{R_{2}}-\frac{1}{R_{1}}\right)
$$

where $\sigma$ is the residual stress of thin films. $E_{\mathrm{s}}$ is Young's modulus of the substrate and $v_{\mathrm{S}}$ is Poisson's ratio of the substrate; for example, $E_{\mathrm{S}}=71.5 \mathrm{GPa}$ and $v_{\mathrm{s}}=0.22$ for B270 glass. $t_{\mathrm{s}}$ is the substrate thickness and $t_{\mathrm{f}}$ the film thickness; $R_{1}$ and $R_{2}$ show the radii of curvature before and after thin film coating.

The anisotropic stress in thin films can be calculated by [20]

$$
\begin{gathered}
\sigma_{x}=\frac{1}{6} \frac{E_{\mathrm{s}}}{1-v_{\mathrm{s}}^{2}}\left(\frac{1}{R_{x}}+\frac{v_{\mathrm{s}}}{R_{y}}\right) \frac{t_{\mathrm{s}}^{2}}{t_{\mathrm{f}}} \\
\sigma_{y}=\frac{1}{6} \frac{E_{\mathrm{s}}}{1-v_{\mathrm{s}}^{2}}\left(\frac{1}{R_{y}}+\frac{v_{\mathrm{s}}}{R_{x}}\right) \frac{t_{\mathrm{s}}^{2}}{t_{\mathrm{f}}}
\end{gathered}
$$

where $\sigma_{x}$ and $\sigma_{y}$ are the biaxial stresses of thin films in the $x$ - and $y$-axis, respectively. $R_{x}$ and $R_{y}$ are the radius of curvature of the film-substrate in the $x$ - and $y$-axis directions, respectively. If $R_{x}$ is equal to $R_{y}$, then Eqs. (2) and (3) can be reduced to Eq. (1). Here the positive sign indicates the tensile stress and the negative sign indicates the compressive stress.

In this work, the average residual stress is calculated from the average curvature using the Stoney formula. For an anisotropic stress evaluation, the deformed shapes of the film-coated substrates are not spherical. To obtain reliable film stress from such aspherical shapes, a Twyman-Green interferometer combined with FFT method are used. The anisotropic stresses in the $x$ - and $y$-axis directions are obtained from the $R_{x}$ and $R_{y}$. The anisotropic residual stress in thin films can be defined in two principal stresses of $\sigma_{x}$ and $\sigma_{y}$. We developed a MATLAB program algorithm to reconstruct 
a three-dimensional surface profile of the thin films and finally to find the biaxial residual stresses.

The influence of the surface roughness on the performance of ITO thin films was also studied. Surface roughness is the level of unevenness of the film surface. Surface roughness of thin films most commonly refers to the variations in the height of the film surface relative to a reference plane. There are two surface roughnesses widely reported for thin films, one is average roughness and the other is root-mean-square (RMS) roughness. They represent the deviation of hillocks and valleys on the film's surface from a reference plane. Average roughness is simply the average of positive hillocks deviation and negative valley deviation values from the reference plane. But the average roughness does not truly reflect the surface deviation. Therefore, we used a home-made Linnik interferometer microscope to determine the RMS surface roughness of thin films, as shown in Fig. 3. For non-contact surface roughness measurement, we present a Linnik microscopic interferometer associated with fast Fourier transform (FFT) algorithm to reconstruct surface topography of thin films [21]. After reconstructing the film's surface, we make use of a Gaussian filter to filter out the high-frequency signal and to obtain roughness profile. This non-contact method was used to measure different rough ITO thin films by the FFT algorithm and digital filter process. In general, the film surface roughness was studied by atomic force microscopy (AFM) in tapping

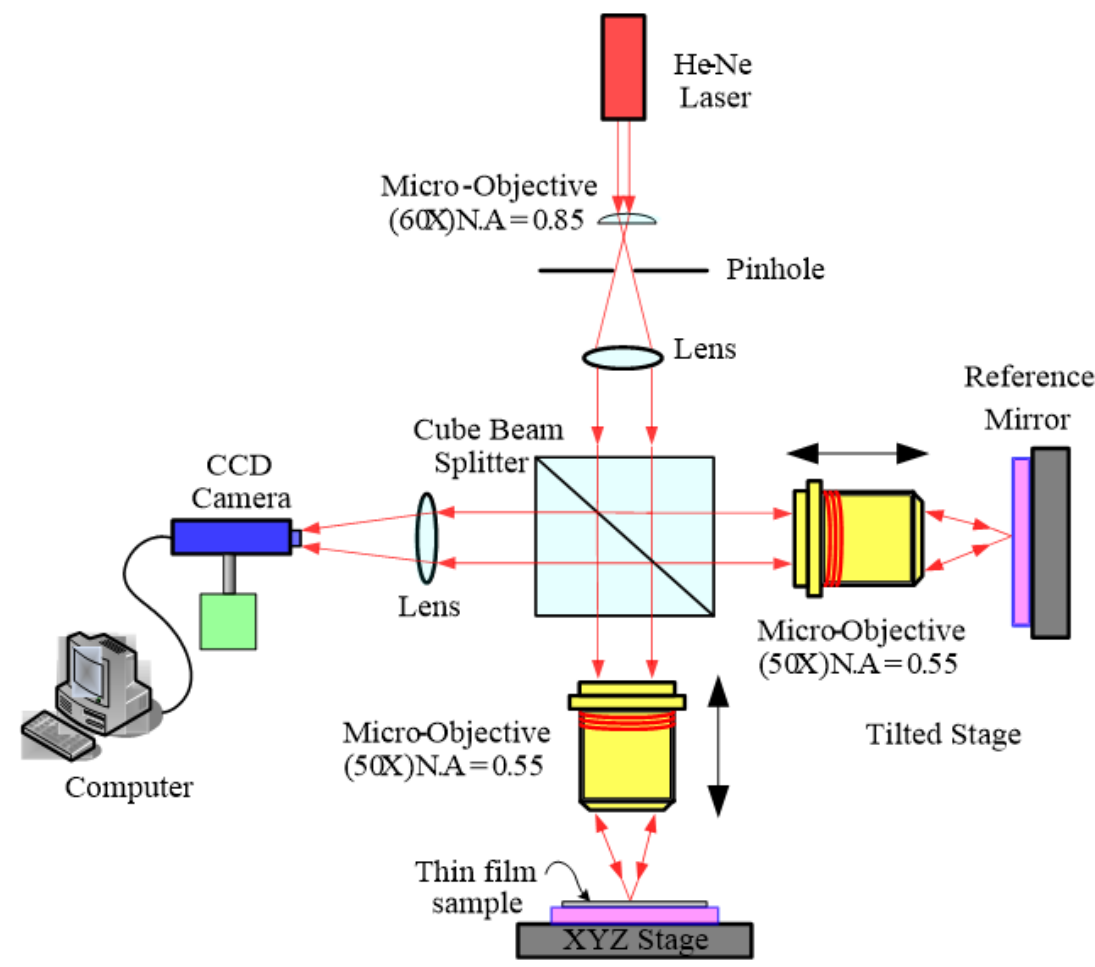

Fig. 3. Schematic representation of a Linnik microscopic interferometer setup. 
-mode $[\underline{22}, 23]$. AFM was also used to confirm the results obtained by means of the proposed method. The surface roughness values were obtained by a home-made Linnik microscopic interferometer. In Fig. 3, a He-Ne laser with the wavelength of $632.8 \mathrm{~nm}$ is used as the light source. The light beam falls on a cube beam-splitter which splits the beam into a reference and a test beam that emerge in perpendicular directions. One beam is used to direct onto a test surface through a cube beam-splitter and a microscope objective $(20 \times)$; another beam is reflected by a reference mirror (flatness $\lambda / 20$ ) and a cube beam-splitter. The test surface within the field of view of the objectives is thus uniformly illuminated and the area of interest on the test surface can be adjusted through alignment of the iris diaphragm. The beams reflected off the reference and the test surface onto a CCD sensor through a lens. The resulting interference fringe pattern is recorded by a CCD camera (Sony; ICX252) and corresponding signal is processed by a computer. This camera has a resolution of $2048 \times 1536$ pixels ( 3.1 megapixel) and the sensor's size is $1 / 1.8^{\prime \prime}$, thus pixel size is $3.45 \mu \mathrm{m} \times 3.45 \mu \mathrm{m}$. The process of image patterns with spatial-carrier phase is carried out with the support of the MATLAB -based software. Only the central $240 \times 240$ pixels, representing an actual dimension of $100 \mu \mathrm{m} \times 100 \mu \mathrm{m}$, were chosen for analysis in order to prevent any edge effects.

A four-point probe apparatus was used to find electrical resistivity of ITO thin film. The four-point probe approach provides more reliable test results. The resistivity and sheet resistance of a thin film can be inferred from the electrical resistance measured. The coating thickness is one of the most important thin-film parameters, since it largely affects the properties of a thin film. The alpha-step profilometer was popularly used to measure the film's thickness by means of the step formed between a film and its substrate. Thus, all film thicknesses were determined by the alpha step D-600 stylus profiler in this study.

\section{Results and discussion}

Various growth techniques and sputtering parameters have an essential affect in controlling the properties of ITO films. This work mainly investigates the effect of the deposited thickness on the electrical and mechanical properties of sputtered ITO films prepared by using RF magnetron sputtering technique.

\subsection{Dependence of the electrical resistivity on film thickness}

The thickness dependence of the electrical conductivity of sputtering ITO film was investigated experimentally. For the resistivity measurement of ITO thin films, the average resistivity values are $1.85 \times 10^{-3} \Omega \cdot \mathrm{cm}$ for $200 \mathrm{~nm}, 1.62 \times 10^{-3} \Omega \cdot \mathrm{cm}$ for $300 \mathrm{~nm}$, and $5.20 \times 10^{-4} \Omega \cdot \mathrm{cm}$ for $350 \mathrm{~nm}$. The lowest resistivity $\left(5.20 \times 10^{-4} \Omega \cdot \mathrm{cm}\right)$ was obtained from ITO films with thickness of $350 \mathrm{~nm}$, as shown in Fig. 4. This measured resistivity value is close to that (from $1.6 \times 10^{-4}$ to $6.5 \times 10^{-4} \Omega \cdot \mathrm{cm}$ ) of the film deposited using similar sputtering method [24-28]. It was observed that the resistivity value decreases with increasing the film thickness from 200 to $350 \mathrm{~nm}$. The variation 


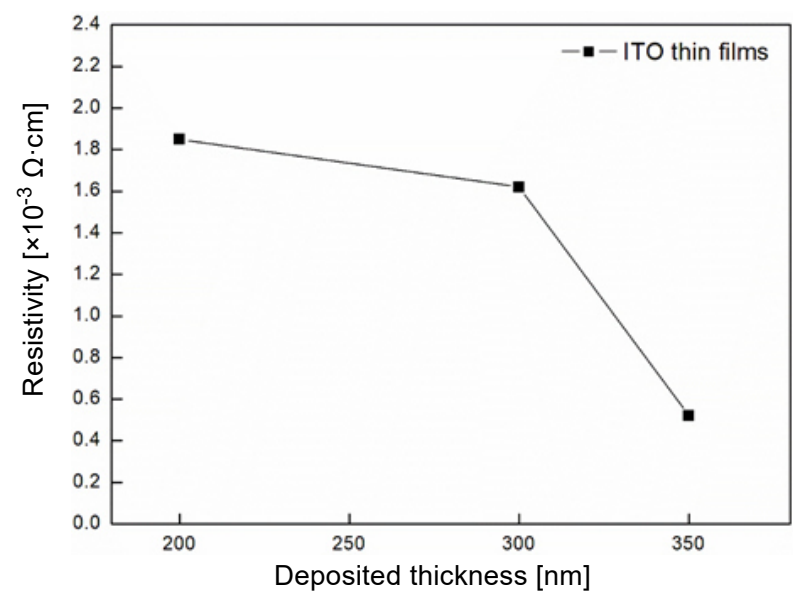

Fig. 4. The average resistivity of ITO films as a function of deposited thickness.

of sheet resistance with the thickness of ITO films is similar to that reported in other studies [29]. The observed dependence of the resistivity on film thickness is in good agreement with the Fuchs-Sondheimer theory [30]. According to Fuchs-Sondheimer theory, some expressions can be derived for the electrical conduction of thin conducting films. The size effect usually occurs on all very thin films, because they usually have an insulating layer or oxide layer on their surface. The resistivity variations were caused by the grain size change in the ITO thin films. The results show that there are significant differences in the electrical resistivity of ITO films with different thicknesses. The decrease of resistivity with increasing the deposited thickness is correlated with the increase in the carrier concentration.

\subsection{Dependence of the residual stress on film thickness}

The biaxial residual stresses in ITO thin films with different deposited thicknesses were investigated experimentally. In order to evaluate the biaxial anisotropic stress of ITO thin films, the surface profile and the radius of curvature were determined by the Twyman-Green interferometer system. Anisotropic stress in thin films exhibits a directional dependence stress in the deposition plane of thin films. In such case, residual stresseses are measured for the directions parallel and perpendicular to the initial deposition plane of the films. Therefore, anisotropic stress state is characterized by two principal stresses. The residual stress in the $x$-axis direction $\sigma_{x}$ and the stress in the $y$ axis direction $\sigma_{y}$. We prepared two groups of ITO samples with different thicknesses. The residual stress measurement results show that the stress anisotropy $\left(\sigma_{x} \neq \sigma_{y}\right)$ is significant and the average compressive stress decreases as the thickness of the films increases. The comparison of the two groups of ITO films was made, and the biaxial anisotropic stress and the average stress vs. film thickness were plotted as shown in Figs. 5 and 6, respectively. It can be seen that when the film thickness increases from 


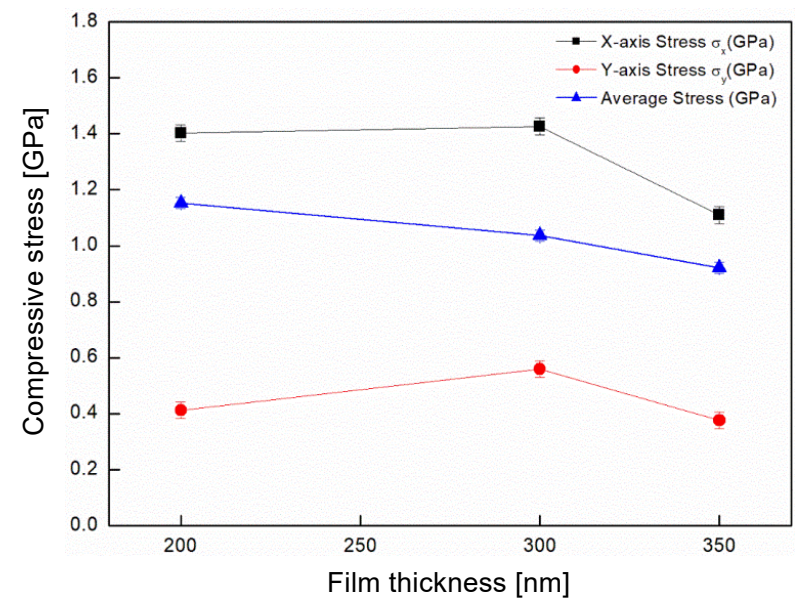

Fig. 5. Biaxial residual stress vs. film thickness for ITO group 1.

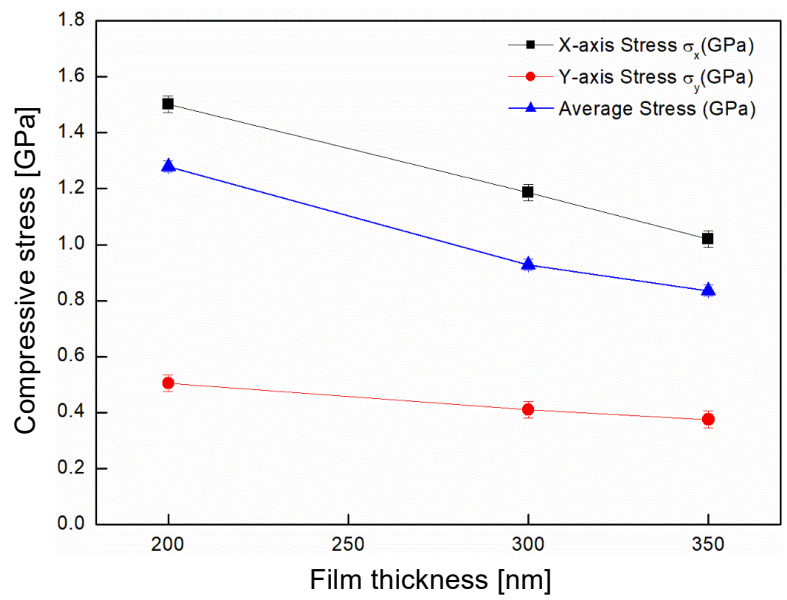

Fig. 6. Biaxial residual stress vs. film thickness for ITO group 2.

200 to $350 \mathrm{~nm}$, the average stress value decreases. Moreover, it should be noted that the two groups of ITO thin films with different thicknesses show a similar tendency in terms of residual stress behavior. The anisotropic stresses in ITO films are found to be more compressive in the $x$-axis direction than that in the $y$-axis direction.

\subsection{Dependence of surface roughness on film thickness}

We evaluated the surface roughness using a homemade Linnik microscopic interferometer (see Fig. 3). Two groups of ITO samples with different thicknesses were measured by the Linnik microscopic interferometer. The roughness measurement is $100 \mu \mathrm{m}$ $\times 100 \mu \mathrm{m}$ in size. RMS value is the square root of the variance or the standard deviation 

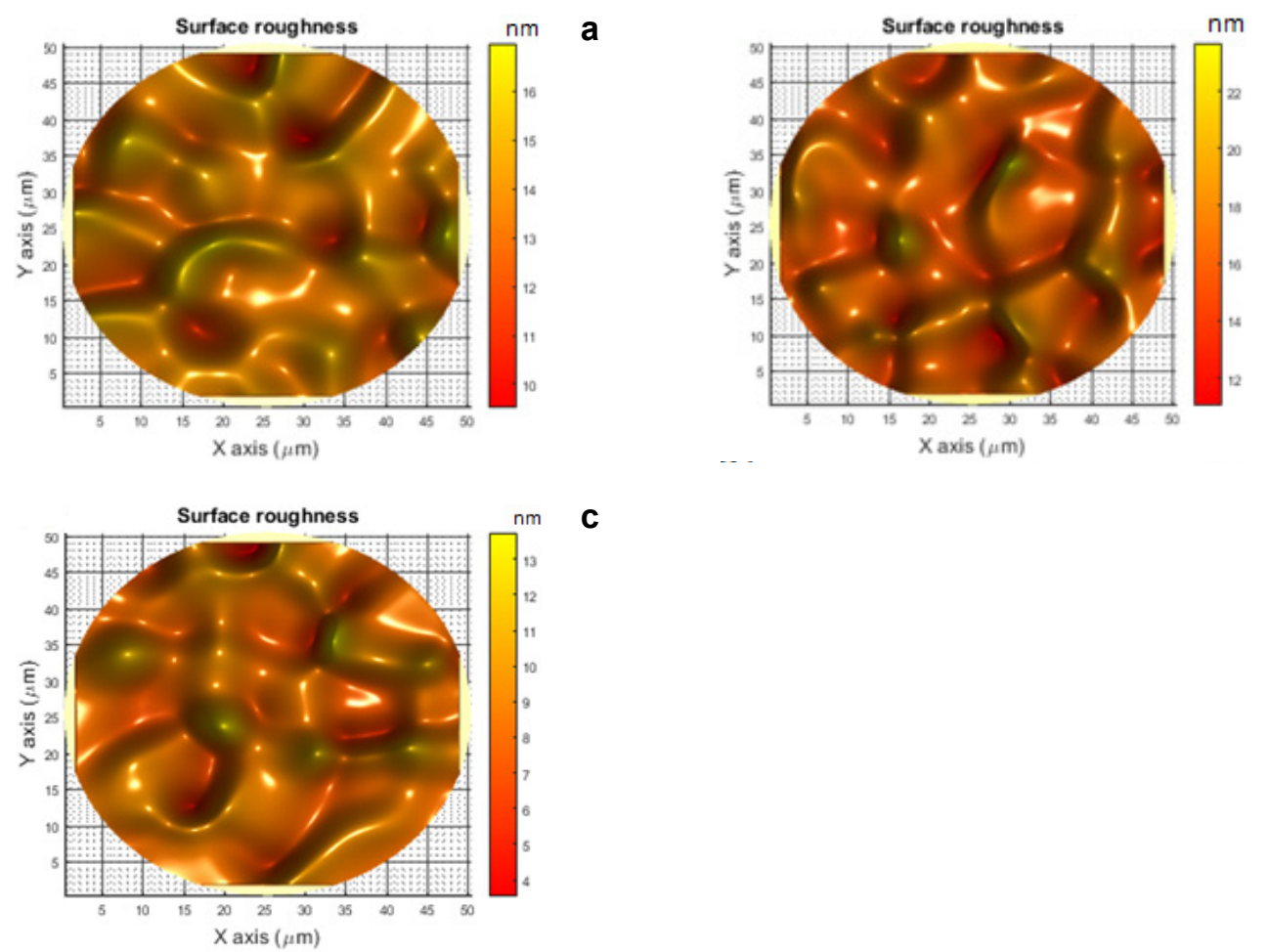

b

Fig. 7. Surface roughness contours of the ITO films deposited with different thicknesses: (a) $200 \mathrm{~nm}$, (b) $300 \mathrm{~nm}$, and (c) $350 \mathrm{~nm}$ (for group 1).

of the film surface from the reference plane within the sampling area. This value is calculated by a statistical formula with 10 measurements. While the film thickness was $300 \mathrm{~nm}$, the RMS surface roughness was $0.874 \pm 0.019 \mathrm{~nm}$ for group 1 and 0.933 $\pm 0.023 \mathrm{~nm}$ for group 2 . However, it slightly decreased when the thickness range was from 300 to $350 \mathrm{~nm}$. The surface morphology of the ITO samples significantly varied with different film thicknesses. Figure 7 reveals the surface roughness contoures of the ITO thin films deposited with different thicknesses for group 1 samples over the scan size of $100 \mu \mathrm{m} \times 100 \mu \mathrm{m}$. The thickness of the thin films is increased by increasing the deposition time. Figure 8 shows the RMS surface roughness as a function of deposited thickness for both groups. The results of roughness measurements for the two groups of ITO samples show a similar tendency. The RMS surface roughness increases gradually from $0.714 \mathrm{~nm}(200 \mathrm{~nm}$ thick film) to $0.874 \mathrm{~nm}$ (300 nm thick film), above which the RMS roughness decreases slightly to $0.852 \mathrm{~nm}$ of the $350 \mathrm{~nm}$ thick film. The stabilization of the surface roughness after certain thickness may attribute to the filling of the holes and craters by the ITO film deposition. As increasing the thickness of ITO thin films, the deposited films were changed from smaller grains to larger grains. In other words, the average grain sizes became larger. 


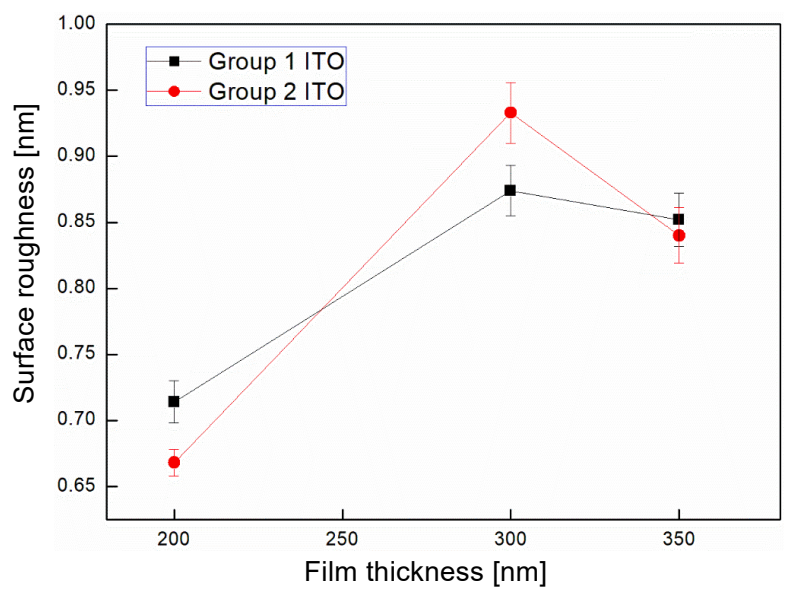

Fig. 8. RMS surface roughness $v s$. film thickness.

Microstructure of ITO thin films was examined by means of field emission scanning electron microscopy (FESEM; Hitachi S-4800). FESEM is an advance technology of SEM because it has a better resolution and more focus beam. The accelerated voltage is ranging from 10 to $40 \mathrm{kV}$ for the SEM. The voltage between cathode and anode is $5 \mathrm{kV}$. The magnification used to scan the thin film was $100 \mathrm{k}$ times. Figure 9 demonstrates the SEM morphologies of ITO thin films prepared by RF magnetron sputtering
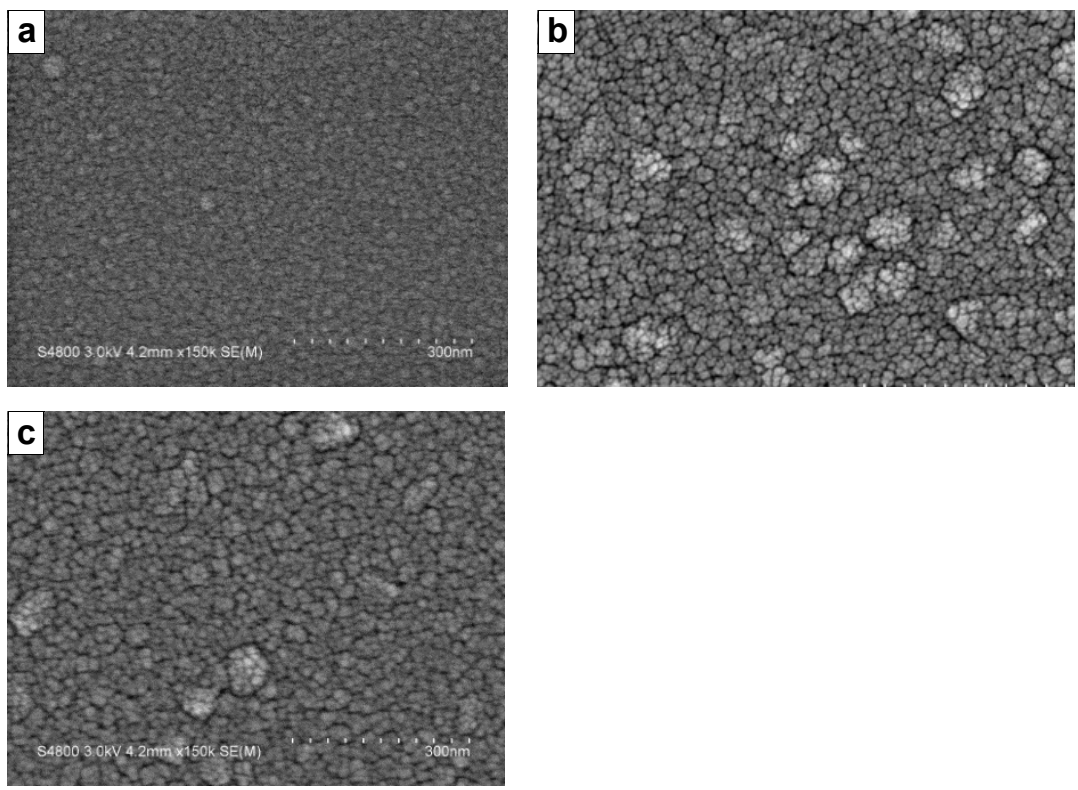

Fig. 9. SEM topography of ITO thin films with different thicknesses: (a) $200 \mathrm{~nm}$, (b) $300 \mathrm{~nm}$, and (c) $350 \mathrm{~nm}$ (for group 1). 

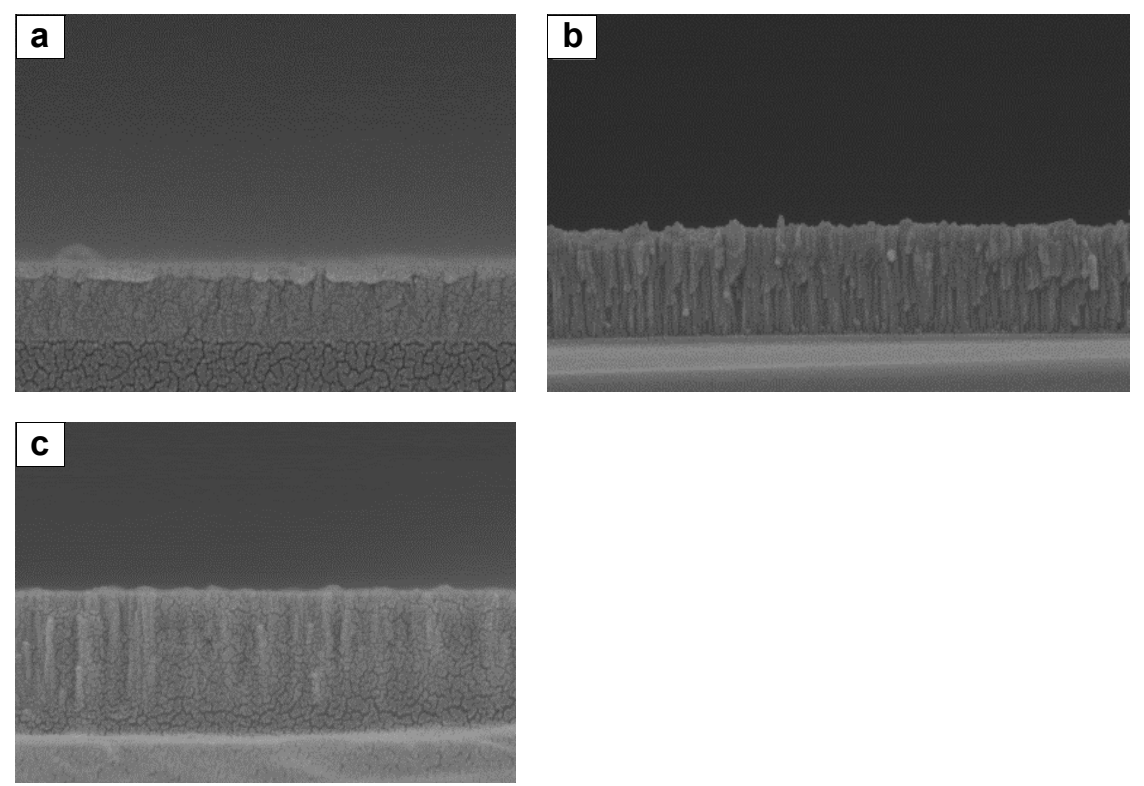

Fig. 10. Cross-sectional SEM micrographs of ITO films with different thicknesses: (a) $200 \mathrm{~nm}$, (b) $300 \mathrm{~nm}$, and (c) $350 \mathrm{~nm}$ (for group 1).

method for different deposited thicknesses. The SEM surface morphologies of ITO thin films reveal a smoother surface, with small grains. It also shows that the grain size increases as the film thickness increased from 200 to $300 \mathrm{~nm}$. A large amount of small grains were observed on the surface of as-deposited ITO films. But the grain size slightly decreases as the film thickness increased from 300 to $350 \mathrm{~nm}$. The results are in agreement with those of surface roughness obtained from the Linnik microscopic interferometer. The cross-sectional SEM micrographs of the ITO thin films are presented in Fig. 10. Almost all the ITO films reveal a compact dense and columnar grains microstructure at different film thicknesses. The grain size slightly increases for ITO films produced from 200 to $350 \mathrm{~nm}$, but some voids are present. The ITO film's microstructure made of compact columnar grains with estimated dimension between 10 and $20 \mathrm{~nm}$.

\section{Conclusions}

We investigated the dependence of resistivity, residual stress and surface roughness on the thickness of sputtering indium tin oxides films grown by a radio-frequency magnetron sputtering system at room temperature. For thin film measurements, the resistivity of ITO films was measured by the four point probe method and the results revealed that the lowest resistivity of $5.20 \times 10^{-4} \Omega \cdot \mathrm{cm}$ was obtained from ITO films with thickness of $350 \mathrm{~nm}$. The mechanical properties of ITO thin films were evaluated 
from the biaxial and the average stress. In general, thin films prepared by sputtering technology are dense in structure and show a compressive stress. Our results show that the compressive stress decreases with increasing the film thickness which results in a decrease in the average stress. We find that the anisotropic stresses in the two groups of ITO films are more compressive in the $x$-direction than those in $y$-direction. The surface roughness measurements for the two groups of ITO thin films with different thicknesses are similar to each other. When the thickness increases, the RMS roughness increases from 0.714 to $0.874 \mathrm{~nm}$, then decreases to $0.853 \mathrm{~nm}$ for ITO group 1, while the value increases from 0.668 to $0.933 \mathrm{~nm}$ and then decreases to $0.840 \mathrm{~nm}$ for ITO group 2. Furthermore, FESEM images demonstrate the smooth surface and morphologies of the ITO films. These results show the RMS surface roughness was varied with ITO film thickness. The electrical resistivity, surface roughness, and residual stress depend strongly on the film thickness which was demonstrated in this work.

Acknowledgment - The authors are grateful for the funding provided by the Ministry of Education of Taiwan (2020 RSC Project No. 20M22026); Ministry of Science and Technology (MOST) of Taiwan under Grands MOST 108-2622-E-035-009-CC3 and 109-2221-E-035-022.

\section{References}

[1] Bender M., Seelig W., Daube C., Frankenberger H., Ocker B., Stollenwerk J., Dependence of oxygen flow on optical and electrical properties of DC-magnetron sputtered ITO films, Thin Solid Films 326(1-2), 1998, pp. 72-77, DOI: 10.1016/S0040-6090(98)00521-5.

[2] SALEHI A., The effects of deposition rate and substrate temperature of ITO thin films on electrical and optical properties, Thin Solid Films 324(1-2), 1998, pp. 214-218, DOI: $\underline{10.1016 / \text { S0040-6090(98) }}$ 00371-X.

[3] You D.J., Chоі S.K., HAN H.S., LeE J.S., Lim C.B., Effect of the deposition geometry on the electrical properties within tin-doped indium oxide film deposited under a given RF magnetron sputtering condition, Thin Solid Films 401(1-2), 2001, pp. 229-234, DOI: 10.1016/S0040-6090(01)01488-2.

[4] Gregory O.J., Luo Q., Crisman E.E., High temperature stability of indium tin oxide thin films, Thin Solid Films 406(1-2), 2002, pp. 286-293, DOI: 10.1016/S0040-6090(01)01773-4.

[5] Morgan D.V., Aliyu Y.H, Bunce R.W., Salehi A., Annealing effects on opto-electronic properties of sputtered and thermally evaporated indium-tin-oxide films, Thin Solid Films 312(1-2), 1998, pp. 268-272, DOI: 10.1016/S0040-6090(97)00733-5.

[6] Bragaglia M., Lamastra F.R., Tului M., Di Gaspare L., Notargiacomo A., Valentini M., Nanni F., Low temperature sputtered ITO on glass and epoxy resin substrates: influence of process parameters and substrate roughness on morphological and electrical properties, Surfaces and Interfaces 17, 2019, article 100365, DOI: 10.1016/j.surfin.2019.100365.

[7] YÜZÜAK D.G., CoșKUN Ö.D., The effect of annealing on the structural, electrical, optical and electrochromic properties of indium-tin-oxide films deposited by RF magnetron sputtering technique, Optik 142, 2017, pp. 320-326, DOI: 10.1016/j.ijleo.2017.06.016.

[8] Zhang K., Zhu F., Huan C.H.A., Wee A.T.S., Effect of hydrogen partial pressure on optoelectronic properties of indium tin oide thin films deposited by radio frequency magnetron sputtering method, Journal of Applied Physics 86(2), 1999, pp. 974-980, DOI: 10.1063/1.370834.

[9] ŢĂLu S., Morozov I.A., YADAV R.P., Multifractal analysis of sputtered indium tin oxide thin film surfaces, Applied Surface Science 484, 2019, pp. 892-898, DOI: 10.1016/j.apsusc.2019.04.170. 
[10] Tien C.L., Lin H.Y., Chang C.K., TANG C.J., Effect of oxygen flow rate on the optical, electrical, and mechanical properties of DC sputtering ITO thin films, Advances in Condensed Matter Physics, Vol. 2018, 2018, article 2647282, DOI: 10.1155/2018/2647282.

[11] Yeom H.Y., Popovich N., Chason E., Paine D.C., A study of the effect of process oxygen on stress evolution in d.c. magnetron-deposited tin-doped indium oxide, Thin Solid Films 411(1), 2002, pp. 17 -22, DOI: 10.1016/S0040-6090(02)00166-9.

[12] Ţălu Ş., Kulesza S., Bramowicz M., Pringle A.M., Pearce J.M., Murugesan M., Venkatachalapathy V., Mayandi J., Micromorphology analysis of sputtered indium tin oxide fabricated with variable ambient combinations, Materials Letters 220, 2018, pp. 169-171, DOI: 10.1016/j.matlet.2018.03.005.

[13] Laux S., Kaiser N., Zöller A. Götzelmann R., Lauth H., Bernitzki H., Room-temperature deposition of indium tin oxide thin films with plasma ion-assisted evaporation, Thin Solid Films 335(1-2), 1998, pp. 1-5, DOI: 10.1016/S0040-6090(98)00861-X.

[14] Narasimha R.K., Optical and electrical properties of indium-tin oxide films, Indian Journal of Pure and Applied Physics 42(3), 2004, pp. 201-204.

[15] Tahar R.B.H., Ban T., Ohya Y., TAKahashi Y., Electronic transport in tin-doped indium oxide thin films prepared by sol-gel technique, Journal of Applied Physics 83(4), 1998, pp. 2139-2141, DOI: $10.1063 / 1.366949$.

[16] Takeda M., Ina H., Kobayashi S., Fourier-transform method of fringe-pattern analysis for computer-based topography and interferometry, Journal of the Optical Society of America 72(1), 1982, pp. 156-160, DOI: 10.1364/JOSA.72.000156.

[17] TIEN C.L., ZENG H.D., Measuring residual stress of anisotropic thin film by fast Fourier transform, Optics Express 18(16), 2010, pp. 16594-16600, DOI: 10.1364/OE.18.016594.

[18] Tien C.L., Lin T.W., Tzeng H.D., Jen Y.J., Liu M.C., Temperature-dependent optical and mechanical properties of obliquely deposited $\mathrm{MgF}_{2}$ thin films, Indian Journal of Pure and Applied Physics 52(2), 2014, pp. 117-123.

[19] Stoney G.G., The tension of metallic films deposited by electrolysis, Proceedings of the Royal Society A 82(553), 1909, pp. 172-175, DOI: 10.1098/rspa.1909.0021.

[20] Zhao J.H, Du Y., Morgen M., Ho P.S., Simultaneous measurement of Young's modulus, Poisson ratio, and coefficient of thermal expansion of thin films on substrates, Journal of Applied Physics 87(3), 2000, pp. 1575-1577, DOI: 10.1063/1.372054.

[21] Tien C.L., YANG H.M., Liu M.C., The measurement of surface roughness of optical thin films based on fast Fourier transform, Thin Solid Films 517(17), 2009, pp. 5110-5115, DOI: 10.1016/j.tsf.2009. 03.193.

[22] Ţălu S., Stach S., Mahajan A., Pathak D., Wagner T., Kumar A., Kumar Bedi R., Multifractal analysis of drop-casted copper (II) tetrasulfophthalocyanine film surfaces on the indium tin oxide substrates, Surface and Interface Analysis 46(6), 2014, pp. 393-398, DOI: 10.1002/sia.5492.

[23] ȚăLu Ș., Micro and Nanoscale Characterization of Three Dimensional Surfaces: Basics and Applications, Napoca Star Publishing House, Cluj-Napoca, Romania, 2015.

[24] LeE J. H., Effects of substrate temperature on electrical and optical properties ITO films deposited by r.f. magnetron sputtering, Journal of Electroceramics 23, 2009, pp. 554-558, DOI: $10.1007 /$ $\underline{\text { s10832-008-9539-6. }}$.

[25] Houng B., WANG A., Characterization of indium tin oxide films by RF-assisted DC magnetron sputtering, Applied Surface Science 258(15), 2012, pp. 5593-5598, DOI: 10.1016/j.apsusc.2012.02.030.

[26] Das R., AdhikARy K., RAy S., The role of oxygen and hydrogen partial pressures on structural and optical properties of ITO films deposited by reactive rf-magnetron sputtering, Applied Surface Science 253(14), 2007, pp. 6068-6073, DOI: 10.1016/j.apsusc.2007.01.107.

[27] Stowell M., Müller J., Ruske M., Lutz M., Linz T., RF-superimposed DC and pulsed DC sputtering for deposition of transparent conductive oxides, Thin Solid Films 515(19), 2007, pp. 7654 -7657, DOI: 10.1016/j.tsf.2006.11.166. 
[28] Calnan S., Upadhyaya H.M., Thwaites M.J., Tiwari A.N., Properties of indium tin oxide films deposited using high target utilisation sputtering, Thin Solid Films 515(15), 2007, pp. 6045-6050, DOI: $10.1016 /$ j.tsf.2006.12.063.

[29] Benoy M.D., Mohammed E.M., Suresh Babu M., Binu P.J., Pradeep B., Thickness dependence of the properties of indium tin oxide (ITO) films prepared by activated reactive evaporation, Brazilian Journal of Physics 39(4), 2009, pp. 629-632, DOI: 10.1590/S0103-97332009000600003.

[30] Horváth G., BaAnKuti J., Resistivity increase in thin conducting films considering the size effect, Physica Status Solidi (a) 110(2), 1988, pp. 549-554, DOI: 10.1002/pssa.2211100228.

Received July 30, 2020

in revised form November 11, 2020 